\title{
Interpolatory curl-free wavelets on bounded domains and characterization of Besov spaces
}

Yingchun Jiang

Correspondence: guilinjiang@126. com

School of Mathematics and Computational Science, Guilin University of Electronic Technology, Guilin, 541004, P. R. China

\section{Abstract}

Based on interpolatory Hermite splines on rectangular domains, the interpolatory curl-free wavelets and its duals are first constructed. Then we use it to characterize a class of vector-valued Besov spaces. Finally, the stability of wavelets that we constructed are studied.

MR(2000) Subject Classification: 42C15; 42C40.

Keywords: interpolatory, curl-free, wavelets, bounded domains, Besov spaces

\section{Introduction}

Due to its potential use in many physical problems, like the simulation of incompressible fluids or in electromagnetism, curl-free wavelet bases have been advocated in several articles and most of the study focus on the cases of $R^{2}$ and $R^{3}$ [1-4]. However, it is reasonable to study the corresponding wavelet bases on bounded domains because of some practical use. At the same time, the stability and the characterization of function spaces are also necessary in some applications, such as the adaptive wavelet methods. In recent years, divergence-free and curl-free wavelets on bounded domains begin to be studied [5-8]. In particular, [8] use the truncation method to obtain interpolatory spline wavelets on rectangular domains from [3]. Inspired by this, we mainly study the interpolatory 3D curl-free wavelet bases on the cube and its applications for characterizing the vector-valued Besov spaces.

In Section 2, we first give the construction of interpolatory curl-free wavelets and its duals on the cube. The characterization of a class of vector-valued Besov spaces are given in part 3. Finally, we also study the stability of the corresponding curl-free wavelets.

Now, we begin with some notations and formulae, which will be used later on. Let $\xi_{1}^{+}$ and $\xi_{2}^{+}$stand for two cubic Hermite splines:

$$
\begin{gathered}
\xi_{1}^{+}(x)=:\left(1-3 x^{2}-2 x^{3}\right) \mathcal{X}_{[-1,0)}(x)+\left(1-3 x^{2}+2 x^{3}\right) \mathcal{X}_{[0,1)}(x), \\
\xi_{2}^{+}(x)=:\left(x+2 x^{2}+x^{3}\right) \mathcal{X}_{[-1,0)}(x)+\left(x-2 x^{2}+x^{3}\right) \mathcal{X}_{[0,1)}(x) .
\end{gathered}
$$

Similarly, the quadratic Hermite splines are defined as

$$
\xi_{1}^{-}(x)=:\left(-6 x-6 x^{2}\right) \mathcal{X}_{[-1,0]}, \xi_{2}^{-}(x)=:\left(1+4 x+3 x^{2}\right) \mathcal{X}_{[-1,0]}(x)+\left(1-4 x+3 x^{2}\right) \mathcal{X}_{[0,1)}(x) .
$$


Let

$$
Z_{j}^{0}=:\left\{0,1, \ldots, 2^{j}\right\}, Z_{j}^{1}=:\left\{1,2, \ldots, 2^{j}\right\}, Z_{j}^{2}=:\left\{0,1, \ldots, 2^{j}-1\right\},
$$

and $Z_{j}^{3}=:\left\{1,2, \ldots, 2^{j}-1\right\}$. For each $j \geq j_{0}$, define the scaling functions on $[0,1]$ :

$$
\begin{gathered}
\xi_{m i j, k}^{\Delta,+}=: \xi_{m}^{+}\left(2^{j} x-k\right) \mathcal{X}_{[0,1]}, k \in Z_{j}^{0} ; \\
\xi_{1 ; j, k}^{\Delta,-}=: \xi_{1}^{-}\left(2^{j} x-k\right) \mathcal{X}_{[0,1]}=\xi_{1}^{-}\left(2^{j} x-k\right), k \in Z_{j}^{1} ; \xi_{2 ; j, k}^{\Delta-}=: \xi_{2}^{-}\left(2^{j} x-k\right) \mathcal{X}_{[0,1]}, k \in Z_{j}^{0} .
\end{gathered}
$$

Let $V_{j}^{\Delta,+}=: \operatorname{span}\left\{\xi_{1 ; j, k^{\prime}}^{\Delta,+}, \xi_{2 j, j, k}^{\Delta,+}: k \in Z_{j}^{0}\right\}, V_{j}^{\Delta,-}=: \operatorname{span}\left\{\xi_{1 ; j, k_{1}}^{\Delta,-}, \xi_{2 j, j, k_{2}}^{\Delta,-}: k_{1} \in Z_{j}^{1}, k_{2} \in Z_{j}^{0}\right\}$, then $\left\{V_{j}^{\Delta,+}\right\}$ and $\left\{V_{j}^{\Delta,-}\right\}$ are two MRAs on $L^{2}([0,1])[8]$. The corresponding duals $\widetilde{\xi}_{m, j, k}^{\Delta, \pm}$ are given in the sense of distributions:

$$
\begin{gathered}
\tilde{\xi}_{1}^{+}=: \delta_{0}\left\langle f, \widetilde{\xi}_{1 ; j, k}^{\Delta,+}\right\rangle=f\left(2^{-j} k\right), k \in Z_{j}^{0} ; \tilde{\xi}_{2}^{+}=:-\delta^{\prime}{ }_{0}\left\langle f, \widetilde{\xi}_{2, j, k}^{\Delta,+}\right\rangle=2^{-j} f^{\prime}\left(2^{-j} k\right), k \in Z_{j}^{0} ; \\
\widetilde{\xi}_{1}^{-}=: \mathcal{X}_{[-1,0]},\left\langle f, \widetilde{\xi}_{1, j, k}^{\Delta,-}\right\rangle=2^{j} \int_{2^{-j}(k-1)}^{2^{-j} k} f(x) d x, k \in Z_{j}^{1} ; \widetilde{\xi}_{2}^{-}=: \delta_{0},\left\langle f, \widetilde{\xi}_{2, j, k}^{\Delta,-}\right\rangle=f\left(2^{-j} k\right), k \in Z_{j}^{0} .
\end{gathered}
$$

The inperpolating multi-wavelets $\eta_{m, j, k}^{\Delta, \pm}$ on $[0,1]$ as well as the wavelet spaces are defined by

$$
\eta_{m ; j, k}^{\Delta, \pm}(x)=: \eta_{m ; j, k}^{ \pm}(x) \mathcal{X}_{[0,1]}=\eta_{m ; j, k}^{ \pm}(x), k \in Z_{j}^{2} ; W_{j}^{\Delta, \pm}=: \operatorname{span}\left\{\eta_{m ; j, k}^{\Delta, \pm}: m=1,2, k \in Z_{j}^{2}\right\}
$$

with $\quad \eta_{m}^{+}=: \xi_{m}^{+}(2 \cdot-1), m=1,2 ; \eta_{1}^{-}=: \xi_{1}^{-}(2 \cdot-1)-\xi_{1}^{-}(2 \cdot-2) ; \eta_{2}^{-}=: \xi_{2}^{-}(2 \cdot-1)$.

Here and after, $h_{j, k}(\cdot)=h\left(2^{j} \cdot-k\right)$. The corresponding duals are given by

$$
\begin{gathered}
\tilde{\eta}_{1}^{+}=: \delta_{\frac{1}{2}}-\frac{1}{2} \delta_{0}-\frac{1}{2} \delta_{1}+\frac{1}{8} \delta^{\prime}{ }_{0}-\frac{1}{8} \delta^{\prime}{ }_{1}, \tilde{\eta}_{2}^{+}=: \frac{3}{4} \delta_{0}-\frac{3}{4} \delta_{1}-\frac{1}{2} \delta^{\prime}{ }_{1}-\frac{1}{2} \delta^{\prime}-\frac{1}{8} \delta^{\prime}{ }_{1}, \\
\tilde{\eta}_{1}^{-}=: \mathcal{X}_{\left[0, \frac{1}{2}\right]}-\mathcal{X}_{\left[\frac{1}{2}, 1\right]}-\frac{1}{4} \delta_{0}+\frac{1}{4} \delta_{1}, \quad \tilde{\eta}_{2}^{-}=: \delta_{\frac{1}{2}}+\frac{1}{4} \delta_{0}+\frac{1}{4} \delta_{1}-\frac{3}{2} \mathcal{X}_{[0,1]}
\end{gathered}
$$

and $\left\langle f, \widetilde{\eta}_{m ; j, k}^{\Delta, \pm}\right\rangle=\left\langle f\left(\frac{\cdot+k}{2^{j}}\right), \widetilde{\eta}_{m}^{ \pm}\right\rangle$. Moreover, there is the following differential relations

$$
\begin{aligned}
& \frac{d}{d x} \xi_{1 ; j, k}^{\Delta,+}(x)=2^{j}\left(\xi_{1 ; j, k}^{\Delta,-}(x)-\xi_{1 j, k}^{\Delta,-}\left(x-2^{-j}\right)\right), k \in Z_{j}^{3} ; \frac{d}{d x} \xi_{1 ; j, 0}^{\Delta_{1}+}(x)=-2^{j} \xi_{1 ; j, 1}^{\Delta,-}(x), \\
& \frac{d}{d x} \xi_{1 ; j, 2^{j}}^{\Delta,+}=2^{j} \xi_{1 ; j, 2 j}^{\Delta,-} ; \frac{d}{d x} \xi_{2 ; j, k}^{\Delta_{,}+}=2^{j} \xi_{2 ; j, k}^{\Delta,-}, k \in Z_{j}^{0} ; \frac{d}{d x} \eta_{m, j, k}^{\Delta,+}=2^{j+1} \eta_{m ; j, k^{\prime}}^{\Delta,-} k \in Z_{j}^{2} . \\
& \frac{d}{d x} \widetilde{\xi}_{1 ; j, k}^{\Delta,-}=-2^{j}\left(\frac{d}{d x} \widetilde{\xi}_{1 ; j, k}^{\Delta,+}-\frac{d}{d x} \widetilde{\xi}_{1 ; j, k-1}^{\Delta,+}\right), \frac{d}{d x} \widetilde{\xi}_{2 ; j, k}^{\Delta,-}=-2^{j} \frac{d}{d x} \widetilde{\xi}_{2 ; j, k}^{\Delta,+}
\end{aligned}
$$

\section{Curl-free wavelets on the cube}

For $\vec{u}(x, y, z)=\left(u_{1}, u_{2}, u_{3}\right)^{T}$, the 3D curl-operator is defined as

$$
\operatorname{curl} \vec{u}=\left(\partial_{2} u_{3}-\partial_{3} u_{2}, \partial_{3} u_{1}-\partial_{1} u_{3}, \partial_{1} u_{2}-\partial_{2} u_{1}\right)^{T} .
$$

Let $I \subseteq\{1,2,3\}=: I_{0}$, define scaling functions

$$
\varphi_{m}^{I}\left(x_{1}, x_{2}, x_{3}\right)=: \prod_{v=1}^{3} \xi_{m_{v}, v}^{I}\left(x_{v}\right), m=\left(m_{1}, m_{2}, m_{3}\right)^{T} \in\{1,2\}^{3}
$$

with $\xi_{\mu, v}^{I}=\left\{\begin{array}{c}\xi_{\mu}^{+}, v \in I \\ \xi_{\mu}^{-}, v \notin I .\end{array}\right.$ 
The corresponding wavelets are

$$
\psi_{e, m}^{I}\left(x_{1}, x_{2}, x_{3}\right)=: \prod_{v=1}^{3} \vartheta_{e_{v}, m_{v}, v}^{I}\left(x_{v}\right), e \in E_{3}^{*}, m=\left(m_{1}, m_{2}, m_{3}\right)^{T} \in\{1,2\}^{3} .
$$

Here and after, $E_{3}^{*}$ denotes the non-zero apexes of the unite cube and $\vartheta_{\ell, \mu, v}^{I}=\left\{\begin{array}{l}\xi_{\mu, v^{\prime}}^{I} \ell=0 \\ \eta_{\mu, v^{\prime}}^{I} \ell=1\end{array}\right.$

Let $\varphi_{m ; j, k}^{\Delta, I}=: \varphi_{m ; j, k}^{I} \mathcal{X}_{[0,1]^{3}}$, which is the tensor product of corresponding interpolatory scaling functions on the interval. The corresponding duals are given similarly. Furthermore, define

$$
\vec{\varphi}_{m, i ; j, k}^{\Delta}=: \varphi_{m ; j, k}^{\Delta, I_{0} \backslash\{i\}} \delta_{i}, \vec{V}_{j}^{\Delta}=: \operatorname{span}\left\{\vec{\varphi}_{m, i ; j, k}^{\Delta}: m \in\{1,2\}^{3}, k \in \nabla_{j}, 1 \leq i \leq 3\right\}
$$

and the projection operators:

$$
\vec{\Lambda}_{j}^{\Delta}=: \Lambda_{j}^{\Delta,\{2,3\}} \delta_{1}+\Lambda_{j}^{\Delta,\{1,3\}} \delta_{2}+\Lambda_{j}^{\Delta,\{1,2\}} \delta_{3}, \vec{\Lambda}_{j}^{\Delta, *}=: \Lambda_{j}^{\Delta,\{1\}} \delta_{1}+\Lambda_{j}^{\Delta,\{2\}} \delta_{2}+\Lambda_{j}^{\Delta,\{3\}} \delta_{3} .
$$

Lemma $\quad 2.1 \quad[8]$ For $\quad$ smooth functions

$f:[0,1]^{3} \rightarrow R, \frac{\partial}{\partial x_{i}} \Lambda_{j}^{\Delta, I} f=\Lambda_{j}^{\Delta, I \backslash\{i\}}\left(\frac{\partial f}{\partial x_{i}}\right), i \in I .$.

\section{Proposition}

2.1 .

For

$\vec{f} \in \vec{C}\left(\operatorname{curl} ;[0,1]^{3}\right)=:\left\{\vec{v} \in\left(C\left([0,1]^{3}\right)\right)^{3}: \operatorname{curl} \vec{v} \in\left(C\left([0,1]^{3}\right)\right)^{3}\right\}$, there has $\operatorname{curl}\left(\vec{\Lambda}_{j} \vec{f}\right)=\vec{\Lambda}_{j}^{\Delta, *}(\operatorname{curl} \vec{f})$.

Proof. Note that Lemma 2.1, then

$$
\begin{aligned}
\vec{\Lambda}_{j}^{\Delta, *}(\operatorname{curl} \vec{f})= & \Lambda_{j}^{\Delta,\{1\}}\left(\frac{\partial f_{3}}{\partial x_{2}}-\frac{\partial f_{2}}{\partial x_{3}}\right) \delta_{1}+\Lambda_{j}^{\Delta,\{2\}}\left(\frac{\partial f_{1}}{\partial x_{3}}-\frac{\partial f_{3}}{\partial x_{1}}\right) \delta_{2}+\Lambda_{j}^{\Delta,\{3\}}\left(\frac{\partial f_{2}}{\partial x_{1}}-\frac{\partial f_{1}}{\partial x_{2}}\right) \delta_{3} \\
= & \left(\frac{\partial}{\partial x_{2}} \Lambda_{j}^{\Delta,\{1,2\}} f_{3}-\frac{\partial}{\partial x_{3}} \Delta_{j}^{\Delta,\{1,3\}} f_{2}\right) \delta_{1}+\left(\frac{\partial}{\partial x_{3}} \Lambda_{j}^{\Delta,\{2,3\}} f_{1}-\frac{\partial}{\partial x_{1}} \Lambda_{j}^{\Delta,\{1,2\}} f_{3}\right) \delta_{2} \\
& +\left(\frac{\partial}{\partial x_{1}} \Lambda_{j}^{\Delta,\{1,3\}} f_{2}-\frac{\partial}{\partial x_{2}} \Lambda_{j}^{\Delta,\{2,3\}} f_{1}\right) \delta_{3},
\end{aligned}
$$

which is curl $\left(\vec{\Lambda}_{j}^{\Delta} \vec{f}\right)$ by definition.

Proposition 2.1 is important, because it tells us that $\vec{\Lambda}_{j}^{\Delta}$ keeps curl-free property. In general, vector-valued wavelets and wavelet spaces are given, respectively, by

$$
\vec{\psi}_{e, m, i j, j, k}^{\Delta}=: \psi_{e, m, j, k}^{\Delta, I_{0} \backslash\{i\}} \delta_{i}, \vec{W}_{j}^{\Delta}=: \operatorname{span}\left\{\vec{\psi}_{e, m, i j, k}^{\Delta}: e \in E_{3}^{*}, m \in\{1,2\}^{3}, 1 \leq i \leq 3, k \in \nabla_{j}^{0}\right\} .
$$

For $e \in E_{3}^{*}$ and $m \in\{1,2\}^{3}$, we define

$$
\vec{\psi}_{e, m ; j, k}^{\Delta, c}=: 2^{-j} \operatorname{grad} \psi_{e, m, j, k}^{\Delta, I_{0}}=2^{-j} \frac{\partial}{\partial x_{1}} \psi_{e, m ; j, k}^{\Delta, I_{0}} \delta_{1}+2^{-j} \frac{\partial}{\partial x_{2}} \psi_{e, m ; j, k}^{\Delta, I_{0}} \delta_{2}+2^{-j} \frac{\partial}{\partial x_{3}} \psi_{e, m ; j, k}^{\Delta, I_{0}} \delta_{3}, k \in \nabla_{j}^{1} .
$$

Clearly, curl $\left(\vec{\psi}_{e, m ;, k}^{\Delta, c}\right)=\overrightarrow{0}$

To give a decomposition for $\vec{W}_{j}^{\Delta}$, take

$$
\vec{\psi}_{e, m, i, j, k}^{\Delta, \text { non }}=: \psi_{e, m ; j, k}^{\Delta, I_{0} \backslash\{i\}} \delta_{i}, k \in \nabla_{j}^{2}
$$


for $i \in I_{0} \backslash\left\{i_{e}\right\}$. Here, we choose $i_{e}$ such that $e_{i_{e}}=1$.

Proposition 2.2. The vector-valued function system $\left\{\vec{\psi}_{e, m, j, k_{1}}^{\Delta, c}, \vec{\psi}_{e, m, i, j, k_{2}}^{\Delta, \text { non }}, e \in E_{3}^{*}, m \in\{1,2\}^{3}, i \neq i_{e}, k_{1} \in \nabla_{j}^{1}, k_{2} \in \nabla_{j}^{2}\right\}$ is complete in $\vec{W}_{j}^{\Delta}$.

Proof. It is sufficient to show the statement for $j=0$. Let $\vec{f} \in \vec{W}_{0}^{\Delta}$ satisfy

$$
\left(\vec{f}, \vec{\psi}_{e, m ; 0, k}^{\Delta, c}\right)=\left(\vec{f}, \vec{\psi}_{e, m, i ; 0, k}^{\Delta, \text { non }}\right)=0
$$

for all $\left.e \in E_{3}^{*}, m \in\{1,2\}^{3}, k \in \nabla_{j}^{1} \cup \nabla_{j}^{2}\right\}, 1 \leq i \leq 3$ and $i \neq i_{e}$. Here, the inner product is in $L^{2}\left([0,1]^{3}\right)$. Without loss of generality, one assumes $i_{e}=1$. Then, $\left(\vec{f}, \vec{\psi}_{e, m, i ; 0, k}^{\Delta, \text { non }}\right)=0$ leads to

$$
\left(f_{2}, \psi_{e, m ; 0, k}^{\Delta, I_{0} \backslash\{2\}}\right)=\left(f_{3}, \psi_{e, m ; 0, k}^{\Delta, I_{0} \backslash\{3\}}\right)=0 .
$$

By the definition of $\psi^{I}$ and differential relations (1.1), one knows

$$
\left(f_{2}, \frac{\partial}{\partial x_{2}} \psi_{e, m ; 0, k}^{\Delta, I_{0}}\right)=\left(f_{3}, \frac{\partial}{\partial x_{3}} \psi_{e, m ; 0, k}^{\Delta, I_{0}}\right)=0 .
$$

Moreover, $\left(\vec{f}, \vec{\psi}_{e, m ; 0, k}^{\Delta, c}\right)=0$ reduces to $\left(f_{1}, \frac{\partial}{\partial x_{1}} \psi_{e, m ; 0, k}^{\Delta, I_{0}}\right)=0$. Now, it follows that $\left(f_{1}, \psi_{e, m ; 0, k}^{\Delta, I_{I} \backslash\{1\}}\right)=0$ from $i_{e}=1$. Finally, $\left(\vec{f}, \vec{\psi}_{e, m, i ; 0, k}^{\Delta}\right)=0$ and $\vec{f}=\overrightarrow{0}$ follows from the definition of $\vec{W}_{0}^{\Delta}$.

To give the bi-orthogonal decomposition, we define

$$
\overrightarrow{\widetilde{\psi}}_{e, m, c, k, k}^{\Delta, c}=\frac{1}{2} \widetilde{\psi}_{e, m i j, k}^{\Delta, I_{0} \backslash\left\{i_{e}\right\}} \delta_{i_{e}}
$$

Assume $I=\left\{i, i_{e}, i\right\}$, then

$$
\text { curl } \vec{\psi}_{e, m, i, i j, k}^{\Delta, \text { non }}=\operatorname{curl} \psi_{e, m i j, k}^{\Delta, I_{0} \backslash\{i\}} \delta_{i}=2^{j+1} \varepsilon_{1} \psi_{e, m i j, k}^{\Delta, I_{0} \backslash\left\{i, i_{e}\right\}} \delta_{i^{\prime}}+\varepsilon_{2} \frac{\partial}{\partial x_{i^{\prime}}} \psi_{e, m ; j, k}^{\Delta, I_{0} \backslash\{i\}} \delta_{i_{e}}
$$

with $\left|\varepsilon_{1}\right|=\left|\varepsilon_{2}\right|=1$ and $\varepsilon_{1} \varepsilon_{2}=-1$. Now, define

$$
\underset{\psi_{e, m, i j, k}}{\overrightarrow{\Delta, \text { non }}}=: \frac{1}{2^{j+1}} \operatorname{curl} \varepsilon_{1} \widetilde{\psi}_{e, m, j, k}^{\Delta, I_{I} \backslash\left\{i, i_{e}\right\}} \delta_{i^{\prime}} .
$$

Here, the derivatives are meant in the sense of distributions. Now, we state the main result:

Proposition

2.3 .

The

set

$\left\{\vec{\psi}_{e, m, i, j, k_{1}}^{\Delta, c}, \vec{\psi}_{e, m, i, j, k_{2}}^{\Delta, \text { non }}, e \in E_{3}^{*}, m \in\{1,2\}^{3}, i \neq i_{e}, j \geq j_{0}, k_{1} \in \nabla_{j}^{1}, k_{2} \in \nabla_{j}^{2}\right\}$ is a bi-orthogonal wavelet basis of $L^{2}\left([0,1]^{3}\right)^{3}$ with duals defined in (2.1) and (2.2).

Proof. According to Proposition 2.2, one only need show

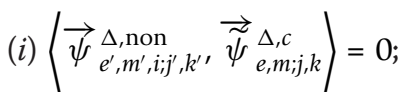

(ii) $\left\langle\vec{\psi}_{e, m i j, k^{\prime}}^{\Delta, c} \underset{\vec{\psi}_{e^{\prime}, m^{\prime}, i j^{\prime}, k^{\prime}}^{\Delta, \text { non }}}{\vec{w}^{\prime}}=0\right.$;

(iii) $\left\langle\vec{\psi}_{e, m, j, k^{\prime}}^{\Delta, c} \underset{\vec{\psi}_{e^{\prime}, m^{\prime} j^{\prime}, k^{\prime}}^{\Delta}}{\Delta}\right\rangle=\delta_{e, e^{\prime}} \delta_{m, m^{\prime}} \delta_{j, j^{\prime}} \delta_{k, k^{\prime}} ;$ 


$$
(i v)\left\langle\vec{\psi}_{e_{1}, m_{1}, i_{1} ; j_{1}, k_{1}}^{\Delta, \text {, }} \underset{\vec{\psi}_{e_{2}, m_{2}, i_{2} ; j_{2}, k_{2}}^{\Delta, \text { non }}}{\vec{x}_{e_{1}, e_{2}} \delta_{m_{1}, m_{2}} \delta_{i_{1}, i_{2}} \delta_{j_{1}, j_{2}} \delta_{k_{1}, k_{2}}}\right.
$$

The identity $(i)$ holds obviously for $i \neq i_{e}$. For $i=i_{e}$, since $i \neq i_{e^{\prime}}$, then $i_{e} \neq i_{e^{\prime}}$, which means $e \neq e^{\prime}$. Finally, the result (i) follows from the bi-orthogonality of $\psi_{e^{\prime}, m^{\prime} j^{\prime}, k^{\prime}}^{\left.\Delta, I^{\prime} \backslash i\right\}}$ and $\widetilde{\psi}_{e, m ; j, k}^{\Delta, I_{0} \backslash\{i\}}$.

Note that $\langle\vec{f}$, curl $\vec{g}\rangle=\langle\operatorname{curl} \vec{f}, \vec{g}\rangle$. Then (ii) follows from curl-grad $=0$. Furthermore,

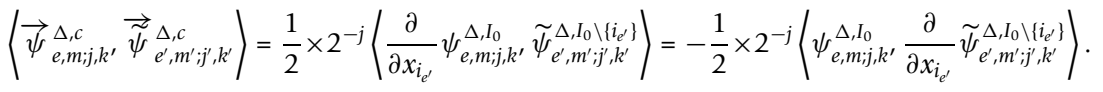

Then by the fact $\frac{d}{d x} \widetilde{\eta}_{m}^{-}=-2 \widetilde{\eta}_{m}^{+}$and the bi-orthogonality of $\psi_{e, m}^{\Delta, I_{0}}, \widetilde{\psi}_{e, m}^{\Delta, I_{0}}$, one obtains

$$
\left\langle\vec{\psi}_{e, m ; j, k^{\prime}}^{\Delta, c} \underset{\Psi_{e^{\prime}, m^{\prime} j^{\prime}, k^{\prime}}^{\Delta, c}}{\overrightarrow{u^{\prime}}}\right\rangle=2^{j^{\prime}-j}\left\langle\psi_{e, m ; j, k^{\prime}}^{\Delta, I_{0}} \widetilde{\psi}_{e^{\prime}, m^{\prime} ; j^{\prime}, k^{\prime}}^{\Delta, I_{0}}\right\rangle=\delta_{e, e^{\prime}} \delta_{m, m^{\prime}} \delta_{j, j^{\prime}} \delta_{k, k^{\prime}}
$$

Now, it remains to prove $(i v)$, which is equivalent to

$$
\begin{gathered}
2^{j_{1}-j_{2}}\left\langle 2 \varepsilon_{1} \psi_{e_{1}, m_{1} ; j_{1}, k_{1}}^{\Delta, I_{1} \backslash\left\{i_{1}, i_{e_{1}}\right\}} \delta_{i^{\prime} 1}+2^{-j_{1}} \varepsilon_{2} \frac{\partial}{\partial x_{i^{\prime}{ }_{1}}} \psi_{e_{1}, m_{1}, j_{1}, k_{1}}^{\Delta, I_{0} \backslash\left\{i_{1}\right\}} \delta_{i_{e_{1}}}, \frac{1}{2} \varepsilon_{1} \widetilde{\psi}_{e_{2}, m_{2}, j_{2}, k_{2}}^{\Delta, I_{2} \backslash\left\{i_{2}, i_{e_{2}}\right\}} \delta_{i^{\prime} 2}\right\rangle \\
=\delta_{e_{1}, e_{2}} \delta_{m_{1}, m_{2}} \delta_{i_{1}, i_{2}} \delta_{j_{1}, j_{2}} \delta_{k_{1}, k_{2}} .
\end{gathered}
$$

It is easily proved, when $e_{1}=e_{2}$ : In fact, since $i_{e_{1}}=i_{e_{2}}$, one can assume $i_{1}=i_{2}$ and $i_{1}^{\prime}=i_{2}^{\prime}$, because $i_{1}=i_{2}^{\prime}$ leads to (2.3) obviously. In that case, the left-hand side of (2.3) reduces to $2 \varepsilon_{1} \cdot \frac{\varepsilon_{1}}{2} \cdot 2^{j_{1}-j_{2}}\left\langle\psi_{e_{1}, m_{1} ; j_{1}, k_{1}}^{\left.I \backslash i_{1}, i_{e}\right\}}, \widetilde{\psi}_{e_{2}, m_{2} ; j_{2}, k_{2}}^{I \backslash\left\{i_{2}, i_{2}\right\}}\right\rangle=\delta_{m_{1}, m_{2}} \delta_{j_{1}, j_{2}} \delta_{k_{1}, k_{2}}$, which is the desired. To the end, it is sufficient to prove that for $e_{1} \neq e_{2}$, that is

$$
\left\langle 2 \varepsilon_{1} \psi_{e_{1}, m_{1} ; j_{1}, k_{1}}^{\Delta, I_{1} \backslash\left\{i_{1}, i_{e_{1}}\right\}} \delta_{i^{\prime}{ }_{1}}+2^{-j_{1}} \varepsilon_{2} \frac{\partial}{\partial x_{i^{\prime} 1}} \psi_{e_{1}, m_{1} ; j_{1}, k_{1}}^{\Delta, I_{1} \backslash\left\{i_{1}\right\}} \delta_{e_{e_{1}}}, \frac{1}{2} \varepsilon_{1} \widetilde{\psi}_{e_{2}, m_{2} ; j_{2}, k_{2}}^{\Delta, I_{2} \backslash\left\{i_{2}, i_{e_{2}}\right\}} \delta_{i^{\prime}{ }_{2}}\right\rangle=0 .
$$

Note that $i_{2}^{\prime} \in\left\{i_{1}, i_{1}^{\prime}, i_{e_{1}}\right\}$. Then the conclusion is obvious when $i_{2}^{\prime}=i_{1}$. When $i_{2}^{\prime}=i_{1}^{\prime}$, then $\left\{i_{1}, i_{e_{1}}\right\}=\left\{i_{2}, i_{e_{2}}\right\}$ and the left-hand side of (2.4) reduces to $2 \varepsilon_{1} \cdot \frac{\varepsilon_{1}}{2}\left\langle\psi_{e_{1}, m_{1}, j, 1}^{\Delta, I_{1} \backslash\left\langle i_{1}, i_{1}\right\}}, \widetilde{\psi}_{e_{2}, m_{2}, j_{2}, k_{2}}^{\left.\Delta, I_{1} \backslash \backslash i_{2}, i_{2}\right\}}\right\rangle=0$. Hence one only need to show (2.4), when $i_{2}^{\prime}=i_{e_{1}}$. However, (2.4) becomes

$$
\left\langle\frac{\partial}{\partial x_{i^{\prime},}} \psi_{e_{1}, m_{1} ; j_{1}, k_{1}}^{\Delta, I_{1} \backslash\left\{i_{1}\right\}}, \widetilde{\psi}_{e_{2}, m_{2} ; j_{2}, k_{2}}^{\Delta, I_{0} \backslash\left\{i_{2}, i_{e_{2}}\right\}}\right\rangle=0 .
$$

in that case. Since $\left\{i_{1}, i_{1}^{\prime}, i_{e_{1}}\right\}=\left\{i_{2}, i_{2}^{\prime}, i_{e_{2}}\right\}=I$, two cases should be considered: $i_{2}=i_{1}, i_{2}^{\prime}=i_{e_{1}}, i_{e_{2}}=i_{1}^{\prime}$ and $i_{2}=i_{1}^{\prime}, i_{2}^{\prime}=i_{e_{1}}, i_{e_{2}}=i_{1}$. Using $\frac{d}{d x} \widetilde{\eta}_{m}^{-}=-2 \widetilde{\eta}_{m}^{+}$, the left-hand side of $(2.5)$ is

$$
-\left\langle\psi_{e_{1}, m_{1}, j_{1}, k_{1},}^{\Delta, I_{0} \backslash\left\{i_{1}\right\}}, \frac{\partial}{\partial x_{i^{\prime} 1}} \widetilde{\psi}_{e_{2}, m_{2}, j_{2}, k_{2}}^{\Delta, I_{2} \backslash\left\{i_{2}, i_{e_{2}}\right\}}\right\rangle=2^{j_{2}+1}\left\langle\psi_{e_{1}, m_{1}, j_{1}, k_{1}}^{\Delta, I_{0} \backslash\left\{i_{1}\right\}}, \widetilde{\psi}_{e_{2}, m_{2} ; j_{2}, k_{2}}^{\Delta, I_{2} \backslash\left\{i_{2}\right\}}\right\rangle=0
$$

in the first case; In the second one, the left-hand side of (2.5) becomes $\left\langle\frac{\partial}{\partial x_{i_{2}}} \psi_{e_{1}, I_{1} ; j_{1}, k_{1}}^{\left.\Delta, I_{1} \backslash i_{1}\right\}} \widetilde{\psi}_{e_{2}, I_{2} ; j_{2}, k_{2}}^{\Delta, I_{0} \backslash\left\{i_{2}, i_{2}\right\}}\right\rangle$. According to the differential relation (1.1), $\frac{\partial}{\partial x_{i_{2}}} \psi_{e_{1}, m_{1} ; j_{1}, k_{1}}^{\left.\Delta, I_{0} \backslash \backslash i_{1}\right\}}$ is 
a linear combination of $\psi_{e_{1}, m_{1}, j_{1}, k_{1}}^{\left.\Delta, I_{1} \backslash i_{1}, i_{2}\right\}}$. By the bi-orthogonality of $\psi_{e_{1}, m_{1}, j_{1}, k_{1}}^{\left.\Delta, I_{1} \backslash i_{1}, i_{2}\right\}}$ and $\widetilde{\psi}_{\left.e_{2}, m_{2}, j_{2}, k_{2}\right\}}^{\Delta \Delta, I_{1} \backslash\left\{i_{1}, i_{2}\right\}}$, one receives the desired conclusion.

\section{Characterization for Besov spaces}

We shall characterize a class of vector-valued Besov spaces in this section. For $0<p, q$ $\leq \infty$ and $s>0$, the Besov space $B_{q}^{s}\left(L^{p}(\Omega)\right)$ is the set of all $f \in L^{p}(\Omega)$ such that

$$
|f|_{B_{q}^{s}\left(L^{p}(\Omega)\right)}=:\left\|\left\{2^{s j} \omega_{m}\left(f, 2^{-j}, L^{p}(\Omega)\right)\right\}\right\|_{\ell^{q}}<+\infty
$$

with $m=[s]+1$ and $\omega_{m}\left(f, 2^{-j}, L^{p}(\Omega)\right)$ the classical $m$-order modulus of smoothness. The corresponding norm is defined by

$$
\|f\|_{B_{q}^{s}\left(L^{p}(\Omega)\right)}=:\|f\|_{L^{p}(\Omega)}+|f|_{B_{q}^{s}\left(L^{p}(\Omega)\right)} .
$$

Our Besov space is defined as

$$
\widehat{B}_{q}^{s}\left(L^{p}\left([0,1]^{3}\right)\right)=:\left\{\vec{f} \in\left(B_{q}^{s}\left(L^{p}\left([0,1]^{3}\right)\right)\right)^{3}: \frac{\partial}{\partial x_{j}} f_{i} \in B_{q}^{s}\left(L^{p}\left([0,1]^{3}\right)\right), i=1,2,3 ; j \neq i\right\}
$$

with the norm

$$
\|\vec{f}\|_{\widehat{B}_{q}^{s}\left(L^{p}\left([0,1]^{3}\right)\right)}=: \sum_{i=1}^{3}\left\|f_{i}\right\|_{B_{q}^{s}\left(L^{p}\left([0,1]^{3}\right)\right)}+\sum_{i=1}^{3} \sum_{\substack{\leq \leq j \leq 3 \\ j \neq i}}\left\|\frac{\partial f_{i}}{\partial x_{j}}\right\|_{B_{q}^{s}\left(L^{p}\left([0,1]^{3}\right)\right)} .
$$

Clearly, curl $\vec{f} \in\left(B_{q}^{s}\left(L^{p}\left([0,1]^{3}\right)\right)\right)^{3}$, when $\vec{f} \in \widehat{B}_{q}^{s}\left(L^{p}\left([0,1]^{3}\right)\right)$.

The following lemma is easily proved by the definition of modulus of smoothness:

Lemma 3.1. If $f(x), g(x) \in B_{q}^{s}\left(L^{p}(R)\right)$, then $f\left(x_{1}\right) g\left(x_{2}\right) \in B_{q}^{s}\left(L^{p}\left(R^{2}\right)\right)$.

For $\alpha=\left(\alpha_{j}\right)_{j \geq j_{0}}$ and $\alpha_{j}=\left(\alpha_{j, k}\right)_{k}$, define

$$
\|\alpha\|_{\ell_{p, q}^{s}}=:\left\|\left(2^{j\left(s-\frac{n}{p}\right)}\left\|\alpha_{j}\right\|_{\ell^{p}}\right)\right\|_{\ell^{q}} .
$$

Lemma 3.2 [8]. If $\phi \in B_{\infty}^{\sigma}\left(L^{p}\left(R^{n}\right)\right)$ is compactly supported, $0<p, q \leq \infty$ and $0<s<\sigma$, then

$$
\begin{aligned}
& \left\|\sum_{k \in \nabla_{j}} \beta_{k} \phi\left(2^{j} \cdot-k\right)\right\|_{B_{q}^{s}\left(L^{p}\left([0,1]^{n}\right)\right)} \lesssim 2^{\left(s-\frac{n}{p}\right) j}\|\beta\|_{\ell^{p},} \\
& \left\|\sum_{j \geq j_{0}} \sum_{k \in \nabla_{j}} \alpha_{j, k} \phi\left(2^{j} \cdot-k\right)\right\|_{B_{q}^{s}\left(L^{p}\left([0,1]^{n}\right)\right)} \lesssim\|\alpha\|_{\ell_{p, q^{s}}}
\end{aligned}
$$

where $\nabla_{j}=:\left\{k: \operatorname{supp} \varphi\left(2^{j} \cdot-k\right) \subseteq[0,1]^{n}\right\}$.

Theorem 3.1. Let $\vec{\varphi}_{m, i, j, k^{\prime}}^{\Delta} \vec{\psi}_{e, m, i j, k^{\prime}}^{\Delta, c}$ and $\vec{\psi}_{e, m, i, j, k}^{\Delta, \text { non }}$ be defined in Section 2. If 0 $<s<1+\frac{1}{p}$ and $0<p, q \leq \infty$, then one has 


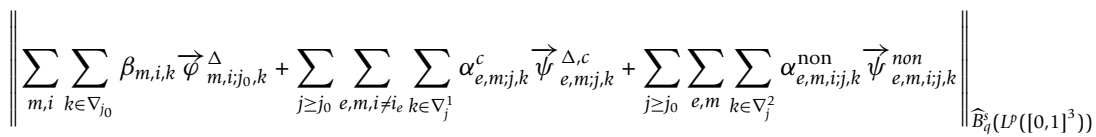

$$
\begin{aligned}
& \lesssim \sum_{m, i}\left\|\beta_{m, i}\right\|_{\ell^{p}}+\sum_{e, m}\left(\sum_{i \neq i_{e}}\left\|\alpha_{e, m, i}^{\mathrm{non}}\right\|_{\ell_{p, q}^{s+1}}+\left\|\alpha_{e, m}^{c}\right\|_{e_{p, q}^{s+1}}\right) .
\end{aligned}
$$

Proof. It is enough to prove the following inequality:

(i)

(i) $\left\|\sum_{k \in \nabla_{j_{0}}} \beta_{m, i, k} \vec{\varphi}_{m, i ; j_{0}, k}^{\Delta}\right\|_{\widehat{B}_{q}^{s}\left(L^{p}\left([0,1]^{3}\right)\right)} \lesssim\left\|\beta_{m, i}\right\|_{\ell p} ;$

(ii) $\left\|\sum_{j \geq j_{0}} \sum_{k \in \nabla_{j}^{1}} \alpha_{e, m ; j, k}^{c} \vec{\psi}_{e, m, j, k}^{\Delta, c}\right\|\left\|_{\widehat{B}_{q}^{s}\left(L^{p}\left([0,1]^{3}\right)\right.} \lesssim\right\| \alpha_{e, m}^{c} \|_{\ell_{p, q}^{s+1 ;}}$

(iii) $\left\|\sum_{j \geq j_{0}} \sum_{k \in \nabla_{j}^{2}} \alpha_{e, m, i, j, k}^{\mathrm{non}} \vec{\psi}_{e, m, i, j, k}^{n o n}\right\|_{\widehat{B}_{q}^{s}\left(L^{p}\left([0,1]^{3}\right)\right.} \lesssim\left\|\alpha_{e, m, i}^{\mathrm{non}}\right\|_{\ell_{p, q}^{s+1}}\left(i \neq i_{e}\right)$.

Let $\vec{h}=: \sum_{k \in \nabla_{j_{0}}} \beta_{m, i, k} \vec{\varphi}_{m, i, j o, k}^{\Delta}$ and $h_{v}$ be the $v$ th component of $\vec{h}$. Then, for $\mu \neq i$,

$$
h_{i}=\sum_{k \in \nabla_{j_{0}}} \beta_{m, i ; k} \varphi_{m}^{I_{0} \backslash\{i\}}\left(2^{j_{0}} x-k\right), \frac{\partial}{\partial x_{\mu}} h_{i}=\sum_{k \in \nabla_{j_{0}}} \beta_{m, i ; k} 2^{j_{0}}\left(\frac{\partial}{\partial x_{\mu}} \varphi_{m}^{I_{0} \backslash\{i\}}\right)\left(2^{j_{0}} x-k\right) .
$$

Since $\xi_{m}^{+} \in B_{\infty}^{2+\frac{1}{p}}\left(L^{p}(R)\right) \subseteq B_{\infty}^{1+\frac{1}{p}}\left(L^{p}(R)\right)$ and $\xi_{m}^{-} \in B_{\infty}^{1+\frac{1}{p}}\left(L^{p}(R)\right)$, then both $\varphi_{m}^{I \backslash\{i\}}$ and $\frac{\partial}{\partial x_{\mu}} \varphi_{m}^{I \backslash\{i\}}$ are in the Besov space ${ }_{B_{\infty}}^{1+\frac{1}{p}}\left(L^{p}\left(R^{3}\right)\right)$, due to Lemma 3.1. Moreover, Lemma 3.2 implies $\left\|h_{i}\right\|_{B_{q}^{s}\left(L^{p}\left([0,1]^{3}\right)\right)} \lesssim\left\|\beta_{m, i}\right\|_{\ell^{p}}$ and $\left\|\frac{\partial}{\partial x_{\mu}} h_{i}\right\|_{B_{q}^{s}\left(L^{p}\left([0,1]^{3}\right)\right)} \lesssim\left\|\beta_{m, i}\right\|_{\ell^{p}}$. Note that $h_{v}=$ 0 for $v \neq i$. Finally, the first inequality follows from the definition.

Let $\vec{g}=: \sum_{j \geq j} \sum_{k \in \nabla_{j}^{1}} \alpha_{e, m i j, k}^{c} \vec{\psi}_{e, m ; j, k}^{\Delta, c}$ and $g_{v}$ be the $v$ th component of $\vec{g}(1 \leq v \leq 3)$. Then

$$
\begin{aligned}
g_{v} & =\sum_{j \geq j_{0}} \sum_{k \in \nabla_{j}^{1}} \alpha_{e, m ; j, k}^{c}\left(\frac{\partial}{\partial x_{v}} \psi_{e, m}^{I_{0}}\right)\left(2^{j} x-k\right), \\
\frac{\partial}{\partial x_{\mu}} g_{v} & =\sum_{j \geq j_{0}} \sum_{k \in \nabla_{j}^{1}} 2^{j} \alpha_{e, m ; j, k}^{c}\left(\frac{\partial^{2}}{\partial x_{\mu} \partial x_{v}} \psi_{e, m}^{I_{0}}\right)\left(2^{j} x-k\right) .
\end{aligned}
$$

Similar to the above, $\frac{\partial}{\partial x_{v}} \psi_{e, m}^{I_{0}} \frac{\partial^{2}}{\partial x_{v} \partial x_{\mu}} \psi_{e, m}^{I_{0}} \in B_{\infty}^{1+\frac{1}{p}}\left(L^{p}\left(R^{3}\right)\right)$. According to Lemma 3.2,

$$
\left\|g_{v}\right\|_{B_{q}^{s}\left(L^{p}\left([0,1]^{3}\right)\right)} \lesssim\left\|\alpha_{e, m}\right\|_{\ell_{p, q}^{s}} \lesssim\left\|\alpha_{e, m}\right\|_{\ell_{p, q}^{s+1}} \text { and } \quad\left\|\frac{\partial}{\partial x_{\mu}} g_{v}\right\|_{B_{q}^{s}\left(L^{p}\left([0,1]^{3}\right)\right)} \lesssim\left\|\alpha_{e, m}\right\|_{\ell_{p, q}^{s+1}} .
$$

Finally, one receives the second inequality and the last one follows analogously. 
Let $W_{\tau}^{\mu}(D)$ denotes the Sobolev space with regularity exponent $\mu$ and domain $D$. Moreover,

$$
E_{d}\left(f, W_{\tau}^{\mu}(D)\right)=: \inf _{P \in \prod_{d-1}}\|f-P\|_{W_{\tau}^{\mu}(D)} .
$$

Furthermore, let $\sigma_{j, k}=2^{-j}\left([0,1]^{3}+k\right)$ for $k \in\left(Z_{j}^{2}\right)^{3}$, the boundary cases are: when there is only one $k_{i}=2^{j}(1 \leq i \leq 3), \sigma_{j, k}$ is defined as replacing the $i$ th position of $2^{-j}$ $\left(\left[k_{1}, k_{1}+1\right] \otimes\left[k_{2}, k_{2}+1\right] \otimes\left[k_{3}, k_{3}+1\right]\right)$ by $\left[2^{j-1}, 2^{j}\right]$; when $k_{i}=k_{i^{\prime}}=2^{i}$ for $i, i^{\prime} \in\{1,2$, $3\}$, both the positions $i$ and $i^{\prime}$ are replaced by $\left[2^{j-1}, 2^{j}\right]$; finally, $\sigma_{j,\left(2^{j}, 2^{j}, 2^{j}\right)}=: 2^{-j}\left(\left[2^{j-1}, 2^{j}\right] \otimes\left[2^{j-1}, 2^{j}\right] \otimes\left[2^{j-1}, 2^{j}\right]\right)$.
Lemma
3.3
Let

$\frac{n}{p}-\frac{n}{\tau}+\mu<s<d, s>0, \mu \in N_{0}, 0<p, q \leq \infty, 1 \leq \tau \leq \infty, j_{0} \in N_{0}$

Then

$$
\left\|\left(2^{j\left(s-\frac{n}{p}+\frac{n}{\tau}-\mu\right)}\left\|\left(E_{d}\left(f, W_{\tau}^{\mu}\left(\sigma_{j, k}\right)\right)\right)_{k \in Z^{n}}\right\|_{\ell p}\right)_{j \geq j_{0}}\right\|_{\ell^{q}} \lesssim|f|_{B_{q}^{s}\left(L^{p}\left([0,1]^{n}\right)\right)} .
$$

The following lemma can be easily proved, but it is important for proving Theorem 3.2:

Lemma 3.4. The following relations hold:

$$
\begin{aligned}
& \left\langle f, \widetilde{\eta}_{1 ; j, k}^{\Delta,+}\right\rangle=f\left(2^{-j}\left(k+\frac{1}{2}\right)\right)-\frac{1}{2} f\left(2^{-j} k\right)-\frac{1}{2}\left(2^{-j}(k+1)\right)-\frac{1}{8} \cdot 2^{-j} f^{\prime}\left(2^{-j} k\right)+\frac{1}{8} \cdot 2^{-j} f^{\prime}\left(2^{-j}(k+1)\right) ; \\
& \left\langle f, \widetilde{\eta}_{2 j, j, k}^{\Delta,+}\right\rangle=2^{-(j+1)} f^{\prime}\left(2^{-j}\left(k+\frac{1}{2}\right)\right)-\frac{3}{4} f\left(2^{-j}(k+1)\right)+\frac{3}{4} f\left(2^{-j} k\right)+\frac{1}{4} \cdot 2^{-(j+1)}\left[f^{\prime}\left(2^{-j} k\right)+f^{\prime}\left(2^{-j}(k+1)\right)\right] ; \\
& \left\langle f, \widetilde{\eta}_{1 ; j, k}^{\Delta-,}\right\rangle=2^{j} \int_{2^{-j} k}^{2^{-j}\left(k+\frac{1}{2}\right)} f(t) d t-2^{j} \int_{2^{-j}\left(k+\frac{1}{2}\right)}^{2^{-j}(k+1)} f(t) d t-\frac{1}{4} f\left(2^{-j} k\right)+\frac{1}{4} f\left(2^{-j}(k+1)\right) ; \\
& \left\langle f, \widetilde{\eta}_{2 j, j, k}^{\Delta,-}\right\rangle=-\frac{3}{2} \cdot 2^{j} \int_{2^{-j} k}^{2^{-j}(k+1)} f(t) d t+\frac{1}{4} f\left(2^{-j} k\right)+\frac{1}{4} f\left(2^{-j}(k+1)\right)+f\left(2^{-j}\left(k+\frac{1}{2}\right)\right) .
\end{aligned}
$$

Theorem 3.2. Let $1+\frac{3}{p}<s<3$ and $0<p, q \leq \infty$. Then

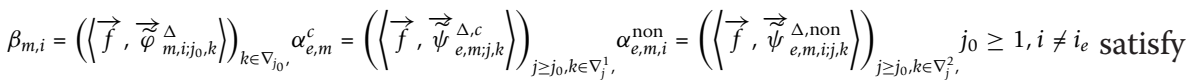

$$
\begin{aligned}
& \sum_{m, i}\left\|\beta_{m, i}\right\|_{\ell^{p}}+\sum_{e, m}\left(\sum_{i \neq i_{e}}\left\|\alpha_{e, m, i}^{\mathrm{non}}\right\|_{\ell_{p, q}^{s+1}}+\left\|\alpha_{e, m}^{c}\right\|_{\ell_{p, q}^{s+q}}\right) \lesssim\|\vec{f}\|_{\widehat{B}_{q}^{s}\left(L^{p}\left([0,1]^{3}\right)\right)}
\end{aligned}
$$

Proof. One only need to show the following inequality:

(i) $\left\|\beta_{m, i}\right\|_{\ell^{p}} \lesssim\|\vec{f}\|_{\widehat{B}_{q}^{s}\left(L^{p}\left([0,1]^{3}\right)\right)}$;

(ii) $\left\|\alpha_{e, m, i}^{\mathrm{non}}\right\|_{\ell_{p, q}^{s+1}} \lesssim\|\vec{f}\|_{\widehat{B}_{q}^{s}\left(L^{p}\left([0,1]^{3}\right)\right)}$;

(iii) $\left\|\alpha_{e, m}^{c}\right\|_{\ell_{p, q}^{s+1}} \lesssim\|\vec{f}\|_{\widehat{B}_{q}^{s}\left(L^{p}\left([0,1]^{3}\right)\right)}$. 
Note that $\beta_{m, i ; k}=\left\langle\vec{f}, \overrightarrow{\widetilde{\varphi}}_{m, i, j_{0}, k}^{\Delta}\right\rangle=\left\langle f_{i}, \widetilde{\varphi}_{m ; j_{0}, k}^{\Delta, I_{0} \backslash\{i\}}\right\rangle$. Then one assumes $i=1$ without loss of generality and proves first

$$
\left\|\beta_{m, 1}\right\|_{\ell^{p}}=\left\|\left(\left\langle f_{1}, \widetilde{\varphi}_{m ; j_{0}, k}^{\Delta,\{2,3\}}\right\rangle\right)_{k \in \nabla^{\prime} j_{0}}\right\|_{\ell^{p}} \lesssim\|\vec{f}\|_{\widehat{B}_{q}^{s}\left(L^{p}\left([0,1]^{3}\right)\right)} .
$$

By the embedding property, $B_{q}^{s}\left(L^{p}(D)\right) \subseteq W_{\tau}^{\mu}(D)$ for $s>\frac{1}{p}-\frac{1}{\tau}+\mu$, and

$$
\|f\|_{W_{\tau}^{\mu}(D)} \lesssim\|f\|_{B_{q}^{s}\left(L^{p}(D)\right)} \cdot
$$

When $m_{2}=m_{3}=1,\left|\left\langle f_{1}, \widetilde{\varphi}_{m_{i j}, k}^{\Delta,\{2,3\}}\right\rangle\right| \leq\left\|f_{1}\right\|_{L^{\infty}\left(\sigma_{j_{0}, k-\delta_{1}}\right)}$ for $m_{1}=1$ or $\left\|f_{1}\right\|_{L^{\infty}\left(\sigma_{\left.j_{0}, k\right)}\right)}$ for $m_{1}=$ 2 .

Using (3.1), one obtains

$$
\left\|\beta_{m, 1}\right\|_{\ell^{p}} \leq \begin{cases}\left(\sum_{k \in \nabla^{\prime} j_{0}}\left\|f_{1}\right\|_{L^{\infty}\left(\sigma_{j_{0}, k-\delta_{1}}\right)}^{p}\right)^{\frac{1}{p}}, m_{1}=1 & \\ \left(\sum_{k \in \nabla^{\prime} j_{0}}\left\|f_{1}\right\|_{L^{\infty}\left(\sigma_{\left.j_{0}, k\right)}\right)}^{p}\right)^{\frac{1}{p}}, m_{1}=2 & \lesssim\left\|f_{1}\right\|_{B_{q}^{s}\left(L^{p}\left([0,1]^{3}\right)\right) \leq}\|\vec{f}\|_{\widehat{B}_{q}^{s}\left(L^{p}([0,1])\right)}\end{cases}
$$

When $m_{3}=2$ (similarly for $m_{2}=2$ ), we obtain

$$
\left|\left\langle f_{1}, \widetilde{\varphi}_{m_{i j}, k}^{\Delta,\{2,3\}}\right\rangle=2^{-j_{0}}\right|\left\langle\frac{\partial f_{1}}{\partial x_{3}}, \widetilde{\varphi}_{m, j_{0}, k}^{\Delta,\{2\}}\right\rangle \mid \leq\left\{\begin{array}{l}
2^{-j_{0}}\left\|\frac{\partial f_{1}}{\partial x_{3}}\right\|_{W_{\infty}^{1}\left(\sigma_{j_{0}, k-\delta_{1}}\right)}, m_{1}=1 \\
2^{-j_{0}}\left\|\frac{\partial f_{1}}{\partial x_{3}}\right\|_{W_{\infty}^{1}\left(\sigma_{\left.j_{0}, k\right)},\right.}, m_{1}=2 .
\end{array}\right.
$$

Note that $1+\frac{1}{p}<s<3$, then the same arguments as above lead to $(i)$.

For $h \in B_{q}^{s}\left(L^{p}\left([0,1]^{3}\right)\right)$, we first claim that there are only the following two cases:

(a) $\mid\left\langle h, \widetilde{\psi}_{e, m, j, k, k}^{\Delta,\left\{i^{\prime}\right\}}\right| \lesssim 2^{-j} E_{3}\left(h, W_{\infty}^{1}\left(\sigma_{j, k}\right)\right)$ or $2^{-j} E_{3}\left(h, W_{\infty}^{1}\left(\sigma_{j, k-\delta_{i}}\right)\right)$;

(b) $\mid\left\langle h, \widetilde{\psi}_{e, m, j, k, k}^{\Delta,\left\{i^{\prime}\right\}}\right| \lesssim E_{3}\left(h, L^{\infty}\left(\sigma_{j, k}\right)\right)$ or $E_{3}\left(h, L^{\infty}\left(\sigma_{j, k-\delta_{i}}\right)\right)$.

In fact, by the vanishing moment property of the dual wavelets, that is,

$$
\tilde{\eta}_{m ; j, k}^{+}\left(P_{1}\right)=0, P_{1} \in \prod_{3} ; \tilde{\eta}_{m ; j, k}^{-}\left(P_{2}\right)=0, P_{2} \in \prod_{2} .
$$

Then $\left\langle h, \widetilde{\psi}_{e, m ; j, k}^{\Delta,\left\{i^{\prime}\right\}}\right\rangle=\left\langle h-P, \widetilde{\psi}_{e, m i j, k}^{\Delta,\left\{i^{\prime}\right\}}\right\rangle$ for each $P \in \Pi_{2}$. Hence, if $e_{i^{\prime}}=1$ or $e_{i^{\prime}}=0$ but $m_{i^{\prime}}$ $=2$, the differential relation (1.1) implies that

$$
\mid\left\langle h, \widetilde{\psi}_{e, m ; j, k}^{\Delta,\left\{i^{\prime}\right\}}|| \lesssim 2^{-j}\left|\left\langle\frac{\partial}{\partial x_{i^{\prime}}}(h-P), \widetilde{\psi}_{e, m ; j, k}^{\Delta, \phi}\right\rangle\right| \lesssim \begin{cases}2^{-j}\|h-P\|_{W_{\infty}^{1}\left(\sigma_{j}, k-\delta_{i}\right)}, e_{i}=0, m_{i}=1 ; \\ 2^{-j}\|h-P\|_{W_{\infty}^{1}\left(\sigma_{j}, k\right),} & \text { o.w. }\end{cases}\right.
$$

Moreover, the (a) part follows from the definition of $E_{3}\left(h, W_{\infty}^{1}(D)\right)$; If $e_{i^{\prime}}=0$ and $m_{i^{\prime}}$ $=1$, 


$$
\mid\left\langle h, \widetilde{\psi}_{e, m, j, k, k}^{\Delta,\left\{i^{\prime}\right\}}|=|\left\langle h-P, \widetilde{\psi}_{e, m, j, k, k}^{\Delta,\left\{i^{\prime}\right\}}\right| \lesssim \begin{cases}\|h-P\|_{L^{\infty}\left(\sigma_{j, k-\delta_{i}}\right)}, & e_{i}=0, m_{i}=1 \\ \|h-P\|_{L^{\infty}\left(\sigma_{j, k}\right)}, & \text { o.w. }\end{cases}\right.
$$

and the $(b)$ part follows.

Now, one is ready to estimate $\alpha_{e, m, i j, k, k}^{\text {non }}$ and $\alpha_{e, m ; j, k}^{c}$. By the definition of $\underset{\psi}{\vec{\psi}, m, i} \stackrel{\Delta, \text { non }}{\text {, one }}$ knows

$$
\begin{aligned}
& \left|\alpha_{e, m, i j, k, k}^{\text {non }}\right|=:\left|\left\langle\vec{f}, \vec{\psi}_{e, m, i, j, k}^{\Delta, \text { non }}\right\rangle=2^{-j-1}\right|\left\langle\operatorname{curl} \vec{f}, \widetilde{\psi}_{e, m, j, k}^{\Delta, I_{0} \backslash\left\{i, i_{e}\right\}} \delta_{i^{\prime}}\right\rangle \mid \\
& =2^{-j-1}\left|\left\langle\frac{\partial f_{i}}{\partial x_{i_{e}}}-\frac{\partial f_{i_{e}}}{\partial x_{i}}, \widetilde{\psi}_{e, m_{j}, k, k}^{\Delta,\left\{i^{\prime}\right\}}\right\rangle\right| \leq 2^{-j-1}\left(\left|\left\langle\frac{\partial f_{i}}{\partial x_{i_{e}}}, \widetilde{\psi}_{e, m, j, k, k}^{\Delta,\left\{i^{\prime}\right\}}\right\rangle\right|+\mid\left\langle\frac{\partial f_{i_{e}}}{\partial x_{i}}, \widetilde{\psi}_{e, m, j, j, k}^{\Delta,\left\{i^{\prime}\right\}}\right\rangle\right) \text {. }
\end{aligned}
$$

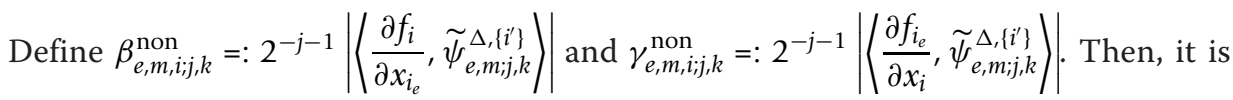
sufficient to show

$$
\left\|\beta_{e, m, i}^{\text {non }}\right\|_{\ell_{p, q}^{s+1}} \lesssim\|\vec{f}\|_{\widehat{B}_{q}^{s}\left(L^{p}\left([0,1]^{3}\right)\right)} \text { and } \quad\left\|\gamma_{e, m, i}^{\text {non }}\right\|_{\ell_{p, q}^{s+1}} \lesssim\|\vec{f}\|_{\widehat{B}_{q}^{s}\left(L^{p}\left([0,1]^{3}\right)\right)} \text {. }
$$

Let $h=: \frac{\partial f_{i}}{\partial x_{i_{e}}}$ in our claim. Then $\beta_{e, m, i j, j, k}^{\text {non }} \lesssim 2^{-2 j} E_{3}\left(h, W_{\infty}^{1}\left(\sigma_{j, k}\right)\right), 2^{-2 j} E_{3}\left(h, W_{\infty}^{1}\left(\sigma_{j, k-\delta_{i}}\right)\right)$ or $\beta_{e, m, i j, j, k}^{\text {non }} \lesssim 2^{-j} E_{3}\left(h, L^{\infty}\left(\sigma_{j, k}\right)\right), 2^{-j} E_{3}\left(h, L^{\infty}\left(\sigma_{j, k-\delta_{i}}\right)\right)$. By $\left.\|\alpha\|_{\ell_{p, q}^{s+1}}=:\left\|2^{j\left(s+1-\frac{n}{p}\right)}\right\| \alpha_{j} \|_{\ell^{p}}\right) \|_{\ell^{q}}$ and Lemma 3.3, one receives that

$$
\left\|\beta_{e, m, i}^{\text {non }}\right\|_{\ell_{p, q}^{s+1}} \lesssim\left|\frac{\partial f_{i}}{\partial x_{i_{e}}}\right|_{B_{q}^{s}\left(L^{p}\left([0,1]^{3}\right)\right)} \lesssim\|\vec{f}\|_{\widehat{B}_{q}^{s}\left(L^{p}\left([0,1]^{3}\right)\right)}
$$

Similarly, $\left\|\gamma_{e, m, i}^{\text {non }}\right\|_{\ell_{p, q}^{s+1}} \lesssim\|\vec{f}\|_{\widehat{B}_{q}^{s}\left(L^{p}\left([0,1]^{3}\right)\right)}$ holds and $\left\|\alpha_{e, m, i}^{\text {non }}\right\|_{\ell_{p, q}^{s+1}} \lesssim\|\vec{f}\|_{\widehat{B}_{q}^{s}\left(L^{p}\left([0,1]^{3}\right)\right)}$.

Finally, to estimate $\alpha_{e, m ; j, k}^{c}=:\left\langle\vec{f}, \vec{\psi}_{e, m, i, k}^{\Delta, c}\right\rangle=\frac{1}{2}\left\langle f_{i_{e}}, \widetilde{\psi}_{e, m ; j, k}^{\Delta, I_{I} \backslash\left\{i_{e}\right\}}\right\rangle$, one assumes without loss of generality that $i_{e}=1$ and $\alpha_{e, m ; j, k}^{c}=\frac{1}{2}\left\langle f_{1}, \widetilde{\psi}_{e, m ; j, k}^{\Delta,\{2,3\}}\right\rangle$. Note that $\frac{d}{d x} \widetilde{\eta}_{m}^{-}=-2 \widetilde{\eta}_{m}^{+}$and $\frac{d}{d x} \widetilde{\xi}_{2}^{-}=-\widetilde{\xi}_{2}^{+}$. Then $\left|\alpha_{e, m ; j, k}^{c}\right| \lesssim 2^{-j}\left|\left\langle\frac{\partial f_{1}}{\partial x_{i}}, \widetilde{\psi}_{e, m i j, k}^{\Delta, l l}\right\rangle\right|$ with $i, l \in\{2,3\}$ and $i \neq l$, when $e_{2}=1$ or $e_{3}=1$ or $m_{2}=2$ or $m_{3}=2$. Similar to the last case, one obtains

$$
\left|\alpha_{e, m ; j, k}^{c}\right| \lesssim 2^{-2 j} E_{3}\left(\frac{\partial f_{1}}{\partial x_{i}}, W_{\infty}^{1}\left(\sigma_{j, k}\right)\right) \quad \text { or } \quad 2^{-j} E_{3}\left(\frac{\partial f_{1}}{\partial x_{i}}, L^{\infty}\left(\sigma_{j, k}\right)\right)
$$

and (iii) is proved in these cases. Now it remains to show (iii), when $e_{2}=e_{3}=0$ and $m_{2}=m_{3}=1$ :

For each $P \in \Pi_{2}$, let $g\left(x_{1},, x_{3}\right)$ be a primitive of $P\left(x_{1}, x_{3}\right)$, i.e.

$$
g\left(x_{1}, x_{2}, x_{3}\right)=: \int P\left(x_{1}, x_{2}, x_{3}\right) d x_{2},
$$

moreover, $\left.g\right|_{x_{2}=2^{-j}\left(k_{2}-1\right)}=:\left.f_{1}\right|_{x_{2}=2^{-j}\left(k_{2}-1\right)}$ if $k_{2}=1,2, \ldots, 2^{j}$ and $\left.g\right|_{x_{2}=2^{-j}}=:\left.f_{1}\right|_{x_{2}=2^{-j}}$ if $k_{2}=$ 0 . Since $e_{1}=1$ and $\widetilde{\eta}_{m}^{-}$has vanishing moments of order 2 , then we obtain 


$$
\begin{aligned}
& \left|\left\langle f_{1}-g, \widetilde{\psi}_{e, m ; j, k}^{\Delta,\{2,3\}}\right\rangle\right|=\left\{\begin{array}{l}
\mid-2^{-j}\left\langle\left(f_{1}-g\right), \frac{\partial}{\partial x_{2}} \widetilde{\psi}_{e, m, j, k}^{\Delta,\{3\}}\right\rangle+\left\langle f_{1}-g, \widetilde{\psi}_{e, m ; j, k-\delta_{2}}^{\Delta,\{2,3\}}\right\rangle, \quad k_{2}=1,2, \ldots, 2^{j} ; \\
2^{-j}\left\langle\left(f_{1}-g\right), \frac{\partial}{\partial x_{2}} \widetilde{\psi}_{e, m, j, k+\delta_{2}}^{\Delta,\{3\}}\right\rangle+\left\langle f_{1}-g, \widetilde{\psi}_{e, m, j, k+\delta_{2}}^{\Delta,\{2,3\}}\right\rangle \mid, \quad k_{2}=0
\end{array}\right. \\
& =\left\{\begin{array}{lc}
-2^{-j}\left\langle\left(f_{1}-g\right), \frac{\partial}{\partial x_{2}} \widetilde{\psi}_{e, m, j, k}^{\Delta,\{3\}}\right\rangle, & k_{2}=1,2, \ldots, 2^{j} ; \\
2^{-j}\left\langle\left(f_{1}-g\right), \frac{\partial}{\partial x_{2}} \widetilde{\psi}_{e, m, j, k+\delta_{2}}^{\Delta,\{3\}}\right\rangle, & k_{2}=0
\end{array}\right. \\
& \lesssim\left\{\begin{array}{l}
2^{-j}\left\|\frac{\partial f_{1}}{\partial x_{2}}-\frac{\partial g}{\partial x_{2}}\right\|_{L^{\infty}\left(\sigma_{j, k-\delta_{2}}\right)}, k_{2}=1,2, \ldots, 2^{j} ; \\
2^{-j}\left\|\frac{\partial f_{1}}{\partial x_{2}}-\frac{\partial g}{\partial x_{2}}\right\|_{L^{\infty}\left(\sigma_{j, k}\right)}, \quad k_{2}=0
\end{array}\right. \\
& =\left\{\begin{array}{l|l}
2^{-j}\left\|\frac{\partial f_{1}}{\partial x_{2}}-P\right\|_{L^{\infty}\left(\sigma_{j, k-\delta_{2}}\right)}, & k_{2}=1,2, \ldots, 2^{j} ; \\
2^{-j}\left\|\frac{\partial f_{1}}{\partial x_{2}}-P\right\|_{L^{\infty}\left(\sigma_{j, k}\right)}, \quad k_{2}=0 .
\end{array}\right.
\end{aligned}
$$

Therefore, we have

$$
\left|\left\langle f_{1}, \widetilde{\psi}_{e, m, j, k}^{\Delta,\{2,3\}}\right\rangle\right|=\left|\left\langle f_{1}-g, \widetilde{\psi}_{e, m ; j, k}^{\Delta,\{2,3\}}\right\rangle\right| \lesssim\left\{\begin{array}{l}
2^{-j} E_{3}\left(\frac{\partial f_{1}}{\partial x_{2}}, L^{\infty}\left(\sigma_{j, k-\delta_{2}}\right)\right), k_{2}=1,2, \ldots, 2^{j} \\
2^{-j} E_{3}\left(\frac{\partial f_{1}}{\partial x_{2}}, L^{\infty}\left(\sigma_{j, k}\right)\right), \quad k_{2}=0 .
\end{array}\right.
$$

The desired result follows from Lemma 3.3.

It should be pointed out that there is no common range for $s$ in Theorems 3.1 and 3.2. Indeed, this is a big shortcoming. However, we need only one estimate in many cases.

\section{The stability of curl-free wavelet bases}

In this part, we shall prove that the single-scale wavelet bases that we have constructed in Section 2 are stable. The following lemma is the classical result of functional analysis:

Lemma 4.1. Let $X$ be a Banach space and $x_{1}, x_{2}, \ldots, x_{n} \subseteq X$ be linearly independent. Then there exists a constant $C>0$ such that for any scalars $\alpha_{1}, \alpha_{2}, \ldots, \alpha_{n}$, one has

$$
\left\|\alpha_{1} x_{1}+\alpha_{2} x_{2}+\ldots+\alpha_{n} x_{n}\right\| \geq C\left(\left|\alpha_{1}\right|+\left|\alpha_{2}\right|+\ldots+\left|\alpha_{n}\right|\right) .
$$

Lemma 4.2 [8]. Let $X$ be a Banach space and $f_{i 1}, f_{i 2}, \ldots, i n_{i} \subseteq X$ be linearly independent for each $i=1,2, \ldots, m$, then the tensor products $\left\{f_{1 j_{1}}\left(x_{1}\right) f_{2 j_{2}}\left(x_{2}\right) \ldots f_{m j_{m}}\left(x_{m}\right)\right\} j_{i} \in\left\{1,2, \ldots, n_{i}\right\}, i=1,2, \ldots, m$ are also linearly independent.

Theorem 4.1. The function system $\left\{2^{\frac{3 j}{2}} \vec{\psi}_{\substack{\Delta, m, c j, k^{\prime} \\ \Delta}} 2^{\frac{3 j}{2}} \vec{\psi}_{e, m, i, j, k^{\prime}}^{\Delta, \text { non }}, e \in E_{3}^{*}, m \in\{1,2\}^{3}, k \in \nabla_{j}^{1}, k^{\prime} \in \nabla_{j}^{2}, i \neq i_{e}\right\}$ generates a Riesz basis for $\vec{W}_{j}^{\Delta}$ with Riesz bounds independent of $j$.

Proof. By Proposition 2.2, one need only show the stability of the function system. Let

$$
\vec{\omega}=: \sum_{e, m, k} d_{e, m ; j, k}^{c} \vec{\psi}_{e, m, c, k}^{\Delta, c}+\sum_{e, m, k} \sum_{i \neq i_{e}} d_{e, m, i, j, k}^{\text {non }} \vec{\psi}_{e, m, i, i j, k}^{\Delta, \text { non }} \in \vec{W}_{j}^{\Delta}
$$


Then $\|\vec{\omega}\|_{L^{2}\left([0,1]^{3}\right)^{3}} \leq\|\vec{\omega}\|_{H^{s}\left([0,1]^{3}\right)^{3}}$ for $s>0$. Since $H^{s}\left([0,1]^{3}\right)=B_{2}^{s}\left(L^{2}\left([0,1]^{3}\right)\right)$, then $\|\vec{\omega}\|_{L^{2}\left([0,1]^{3}\right)^{3}} \leq\|\vec{\omega}\|_{\widehat{B}_{2}^{s}\left(L^{2}\left([0,1]^{3}\right)^{3}\right)}$. Moreover, one receives

$$
\|\vec{\omega}\|_{L^{2}\left([0,1]^{3}\right)^{3}} \lesssim\left(\sum_{e, m, k} \sum_{i \neq i_{e}}\left|d_{e, m, i j, j, k}^{\mathrm{non}}\right|^{2}+\sum_{e, m, k}\left|d_{e, m i j, k}^{c}\right|^{2}\right)^{\frac{1}{2}},
$$

due to Theorem 3.1. Now, it remains to prove the lower bound. Let $\sigma_{j, k}=: 2^{-j}\left([0,1]^{3}+k\right), k \in\left(Z_{j}^{2}\right)^{3}$, then $\bigcup_{k \in\left(Z_{j}^{2}\right)^{3}} \sigma_{j, k}=[0,1]^{3}$. We take an example for $e=$ $(0,0,1)$ and $m=(2,1,1)$,

$$
\begin{aligned}
& \vec{\psi}_{e, m i j, k}^{\Delta, c}=\left(\xi_{2 ; j, k_{1}}^{\Delta,-} \xi_{1 ; j, k_{2}}^{\Delta,+} \eta_{1 ; j, k_{3}}^{\Delta,+} \xi_{2, j, k_{1}}^{\Delta,+}\left(\xi_{1 ; j, k_{2}}^{\Delta,-}-\xi_{1 ; j, k_{2}+1}^{\Delta,-}\right) \eta_{1 ; j, k_{3}}^{\Delta,+}, \xi_{2 ; j, k_{1}}^{\Delta,+} \xi_{1 ; j, k_{2}}^{\Delta,+} \eta_{1 ; j, k_{3}}^{\Delta,-}\right), \\
& \vec{\psi}_{e, m, 1 ; j, k}^{\Delta, \text { non }}=\left(\xi_{2 ; j, k_{1}}^{\Delta,-} \xi_{1 ; j, k_{2}}^{\Delta,+} \eta_{1 ; j, k_{3}}^{\Delta,+} 0,0\right), \vec{\psi}_{e, m, 2 ; j, k}^{\Delta, \text { non }}=\left(0, \xi_{2 ; j, k_{1}}^{\Delta,+} \xi_{1 ; j, k_{2}}^{\Delta,-} \eta_{1 ; j, k_{3}}^{\Delta,+} 0\right) .
\end{aligned}
$$

For each fixed $k \in\left(Z_{j}^{2}\right)^{3}$, by the characteristics of supports, Lemma 4.1 and 4.2 , one has

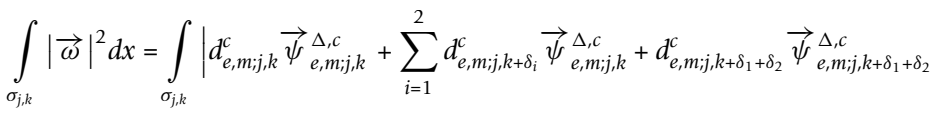

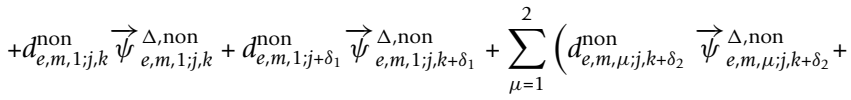

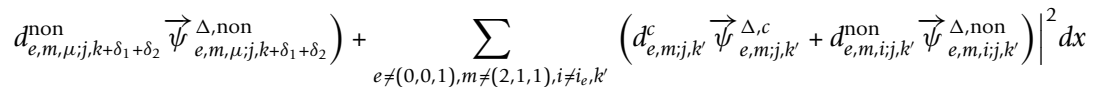

$$
\begin{aligned}
& \geq C\left(\left|d_{e, m ; j, k}^{c}\right|^{2}+\sum_{i=1}^{2}\left|d_{e, m ; j, k+\delta_{i}}^{c}\right|^{2}+\left|d_{e, m ; j, k+\delta_{1}+\delta_{2}}^{c}\right|^{2}+\left|d_{e, m, 1 ; j, k}^{\mathrm{non}}\right|^{2}+\left|d_{e, m, 1 ; j, k+\delta_{1}}^{\text {non }}\right|^{2}\right. \\
& \left.+\sum_{\mu=1}^{2}\left(\left|d_{e, m, m, j ;, k+\delta_{2}}^{\mathrm{non}}\right|^{2}+\left|d_{e, m, \mu, j, k+\delta_{1}+\delta_{2}}^{\mathrm{non}}\right|^{2}\right)+\sum_{e \neq(0,0,1), m \neq(2,1,1), i \neq i i_{e}, k^{\prime}}\left(\left|d_{e, m, j, k^{\prime}}^{c}\right|^{2}+\left|d_{e, m, i, j, k^{\prime}}^{\text {non }}\right|^{2}\right)\right) .
\end{aligned}
$$

Finally, the lower estimation follows from

$$
\|\vec{\omega}\|_{L^{2}\left([0,1]^{3}\right)^{3}}^{2}=\sum_{\left.k \in\left(Z_{j}^{2}\right)^{3}\right)_{j, k}} \int_{j, k}|\vec{\omega}|^{2} d x \gtrsim C\left(\sum_{e, m, k} \sum_{i \neq i_{e}}\left|d_{e, m, i j, j, k}^{\text {non }}\right|^{2}+\sum_{e, m, k}\left|d_{e, m ; j, k}^{c}\right|^{2}\right) .
$$

Corollary 4.2. The system $\left\{2^{\frac{3 j}{2}} \vec{\psi}_{e, m, c, k^{\prime}}^{\Delta, c}, e \in E_{3^{*}}^{*}, m \in\{1,2\}^{3}, k \in \nabla_{j}^{1}\right\}$ is a Riesz basis for $\vec{W}_{j}^{\Delta, c}=: \operatorname{span}\left\{2^{\frac{3 j}{2}} \vec{\psi}_{e, m ; j, k^{\prime}}^{\Delta, c}, e \in E_{3}^{*}, m \in\{1,2\}^{3}, k \in \nabla_{j}^{1}\right\}$ with bounds independent of $j$.

Proof. Note that $\vec{\omega}=\sum_{e, m, k} d_{e, m, j, k}^{c} \vec{\psi}_{e, m, j, k}^{\Delta, c}+\sum_{e, m, k} \sum_{i \neq i_{e}} d_{e, m, i j, k}^{\text {non }} \vec{\psi}_{e, m, i j, j, k}^{\Delta, \text { non }} \in \vec{W}_{j}^{\Delta}$ and curl.grad $=0$. Then the desired result follows from the fact that $\vec{\omega}$ is curl-free if and only if for all $d_{e, m, i, j, k}^{\text {non }}=0$. 


\section{Acknowledgements}

This is supported by the 863 Project of China(No. 2012AA011005), the project of Guangxi Innovative Team(No. 2012jjGAG0001), the National Natural Science Foundation of China (No.11161014, 11001062) and the fund of Education Department of Guangxi (No.201012M9094, 201102ZD015, 201106LX172).

\section{Competing interests}

The authors declare that they have no competing interests.

Received: 27 December 2011 Accepted: 23 March 2012 Published: 23 March 2012

\section{References}

1. Deriaz, E, Perrier, V: Towards a divergence-free wavelet method for the simulation of $2 \mathrm{D} / 3 \mathrm{D}$ turbulent flows. J Turbul. 7(3):37 (2006)

2. Deriaz, E, Perrier, V: Orthogonal Helmholtz decomposition in arbitrary dimension using divergence-free and curl-free wavelets. Appl Comput Harmon Anal. 26(2):249-269 (2009). doi:10.1016/j.acha.2008.06.001

3. Bittner, K, Urban, K: On interpolatory divergence-free wavelets. Math Comput. 76, 903-929 (2007). doi:10.1090/500255718-06-01949-1

4. Urban, K: Wavelet bases in H(div) and H(curl). Math Comput. 70(234):739-766 (2001)

5. Stevenson, R: Divergence-free wavelet bases on the hypercube. Appl Comput Harmon Anal. 30, 1-19 (2011). doi:10.1016/j.acha.2010.01.007

6. Stevenson, R: Divergence-free wavelet bases on the hypercube: Free-slip boundary conditions, and applications for solving the instationary Stokes equations. Math Comput. 80, 1499-1523 (2011). doi:10.1090/S0025-5718-2011-02471-3

7. Harouna, SK, Perrier, V: Divergence-free and curl-free wavelets on the square for numerical simulations. Math Models Methods Appl Sci, Preprint.http://hal.inria.fr/hal-00558474/PDF/perrier-kadri.pdf

8. Zhao, J: Interpolatory Hermite splines on rectangular domains. Appl Math Comput. 216, 2799-2813 (2010). doi:10.1016/j. amc. 2010.03 .130

doi:10.1186/1029-242X-2012-68

Cite this article as: Jiang: Interpolatory curl-free wavelets on bounded domains and characterization of Besov spaces. Journal of Inequalities and Applications 2012 2012:68.

\section{Submit your manuscript to a SpringerOpen ${ }^{\odot}$ journal and benefit from:}

- Convenient online submission

- Rigorous peer review

- Immediate publication on acceptance

- Open access: articles freely available online

- High visibility within the field

- Retaining the copyright to your article

Submit your next manuscript at $\boldsymbol{\wedge}$ springeropen.com 